\title{
Three-dimensional Fourier-domain optical coherence tomography of alveolar mechanics in stepwise inflated and deflated isolated and perfused rabbit lungs
}

\author{
Alexander Krueger*a , Lilla Knels ${ }^{b}$, Sven Meissner ${ }^{a}$, Martina Wendel ${ }^{b}$, Axel R. Heller ${ }^{b}$, Thomas \\ Lambeck $^{\mathrm{c}}$, Thea Koch ${ }^{\mathrm{b}}$, Edmund Koch ${ }^{\mathrm{a}}$ \\ ${ }^{a}$ Clinical Sensoring and Monitoring, Medical Faculty, TU Dresden, Fetscherstr. 74, 01307 Dresden, \\ Germany; \\ ${ }^{b}$ Dept. of Anaesthesiology and Intensive Care, Medical Faculty, TU Dresden, Fetscherstr. 74, 01307 \\ Dresden, Germany; \\ ${ }^{c}$ Dept. of Computer Science, TU Dresden, 01062 Dresden, Germany
}

\begin{abstract}
Fourier domain optical coherence tomography (FD-OCT) was used to acquire three-dimensional image stacks of isolated and perfused rabbit lungs $(n=4)$ at different constant pulmonary airway pressures $(C P A P)$ and during vascular fixation. After despeckling and applying a threshold, the images were segmented into air and tissue, and registered to each other to compensate for movement between CPAP steps. The air-filled cross-sectional areas were quantified using a semiautomatic algorithm. The cross-sectional area of alveolar structures taken at all three perpendicular planes increased with increasing CPAP. Between the minimal CPAP of $3 \mathrm{mbar}$ and the maximum of $25 \mathrm{mbar}$ the areas increased to about $140 \%$ of their initial value. There was no systematic dependency of inflation rate on initial size of the alveolar structure. During the perfusion fixation of the lungs with glutaraldehyde morphometric changes of the alveolar geometry measured with FD-OCT were negligible.
\end{abstract}

Keywords: optical coherence tomography, lung, mechanical ventilation, pulmonary alveolus, glutaraldehyde fixation

\section{INTRODUCTION}

\subsection{Ventilator induced lung injury}

Artificial ventilation puts mechanical stress onto the lung parenchyma ${ }^{1}$. The most vulnerable and delicate part of the lung is the alveolar wall. It consists of the fluid covered epithelial cell layer at the air interface and the inner endothelial cells forming the capillaries. Both cell types are attached to an extracellular matrix enforced by collagen and elastin. Apart from major structural damage of the alveolar wall such as rupture or perforation of the air-blood barrier caused by overextension (barotrauma or volutrauma) there is the concept of biotrauma: Unusual high forces acting on the cellmembrane, on the intracellular matrix, on the cell-cell and on the cell-matrix contacts might lead to a physiological response or even pro-inflammatory signaling of the cells $\mathrm{s}^{2.3}$. These three types of traumata are thought to be the causes for ventilator induced or associated lung injury. In order to find more protective ventilation strategies which reduce or circumvent these traumata, a profound understanding of alveolar mechanics in the sense of quantifying the deformations and associated stressing forces is necessary.

\subsection{Understanding lung mechanics}

Lung edema and recruitment of atelactatic lung areas by ventilator maneuvers can be seen in computed tomography scans ${ }^{4}$. However, there is no non-invasive way to make lung mechanics visible on the sub $100 \mu \mathrm{m}$ alveolar scale. This has lead to a controversy about the nature of alveolar deformations during ventilation. Do alveoli simply expand isotropically, do they unscramble by septal unfolding, do additional alveoli open up or do alveolar ducts and bronchioles take up the tidal volume? All of these types of mechanics can either individually or in combination explain the form of the pressure-volume curve observed for the whole lung. But their corresponding stress-impact on the epithelial cell layer will be very different for each case. If the alveoli are edema filled or the surface active agent is deactivated the situation becomes even more complex, because additional stress might be produced by air pressed through edema ${ }^{5}$. 


\subsection{Imaging alveolar geometry changes}

The experimental methodology in addressing the question of alveolar dynamics has developed from histological investigations of alveolar geometry in fixated lungs $^{6}$ to real-time in-vivo video-microscopy of subpleural lung parenchyma in animal models ${ }^{7}$. The latter provides information in form of bright reflections in the dark field micrographs at a focal plane located somewhere just beneath the pleura. These reflections can be caused by alveolar walls, capillary network in the pleura (corner capillaries), pleura enforcing collagen network, or any liquid-air interfaces (in the case of edema). Thus, interpretation of these images is not unambiguous.

\subsection{Optical coherence tomography of the lung}

Recently, optical coherence tomography (OCT) was used to image the alveolar structure of fixated lung parenchyma and to acquire cross-sectional images perpendicular to the pleura at the ventilated isolated lung model ${ }^{8,9}$. Although a qualitative dependence of the alveolar geometry on ventilator settings could be observed with this method, the crosssectional imaging suffered from the restriction to one sectional plane. This restriction eventually leads to artificial geometrical changes of the air-filled volumes produced by movement of the entire lung parenchyma relative to the sectional plane. Consequently, for quantitative measurements of alveolar geometry changes, three-dimensional information would be of great help. Fortunately, OCT in principle provides three-dimensional information. As a first step towards dynamic real-time OCT volume scans of the ventilated lung, a series of three dimensional image stacks was taken at the stepwise inflated and deflated isolated rabbit lung. The aim of this study was to quantatively relate the different transpulmonary pressures to the corresponding sizes of individual alveolar structures derived from the threedimensional OCT scans.

\section{MATERIAL AND METHODS}

\subsection{Isolated rabbit lungs}

The model of the isolated, perfused and ventilated rabbit lung has been published previously ${ }^{10}$. The lungs were ventilated by pressure-controlled ventilation using a KTR 4 small animal ventilator (Hugo-Sachs, Freiburg, Germany) with a PEEP of $3 \mathrm{mbar}$, the respiratory rate was set at $30 / \mathrm{min}$.. Pressure-volume curves were registered by spirometry using a Fleisch pneumotachograph (Hugo-Sachs). Then, constant airway pressure (CPAP) was applied and stepwise increased up to 25 mbar and decreased again down to 3 mbar. At each CPAP-level $(5,10,15,20,25,20,15,10,5,4,3$ mbar $)$ a 3D-OCT scan was performed.

\subsection{Fourier-domain optical coherence tomography system}

The setup of the OCT system is shown in Fig. 1. The light source is a superluminescence diode (SLD, power: $1 \mathrm{~mW}$, central wavelength $840 \mathrm{~nm}$, full spectral width at half maximum: $50 \mathrm{~nm}$, Superlum, Russia). The light is propagated through a fiber optic coupler to the scanner head, where it is collimated to a free space beam. A 50:50 beam splitter cube splits the light into sample arm and reference arm. The intensity of the reference light can be adjusted by changing the focus position in respect to the reference mirror. The sample light is deflected by two galvanometer driven scanner mirrors, again deflected by a hot mirror, which is transparent for visible light, and finally focused by a lens $(f=25 \mathrm{~mm})$ into the subpleural lung parenchyma. Reference light and backscattered sample light are superimposed, propagated back in the same fibre, collimated and dispersed by a reflection grating. The resulting interference spectrum is focused on a complementary metal oxide semiconductor line detector with 1024 pixel. The readout and analog to digital conversion rate is set to $1.25 \mathrm{MHz}$, resulting in an amplitude-scan rate of $1.21 \mathrm{kHz}$. Three-dimensional OCT image stacks are acquired by progressively scanning an area of $1.2 \mathrm{~mm} \times 1.2 \mathrm{~mm}$ with $300 \times 300$ spectra and on-the-fly-saving to hard disc. The spectral data is processed offline into grey value image stacks representing the logarithmized Fourier amplitudes.

\subsection{Positioning system}

Because the isolated lung hangs freely, a repositioning of the OCT scanner is necessary each time a new CPAP is set. An integrated small video camera helps to find marker dots on the pleura and to realign the OCT scanner head, which is mounted on a ball and socket base with three additional motor driven translation stages. Two perpendicularly orientated 300 pixel wide OCT brightness-scans are continuously displayed in real-time as an additional help for finding the right position within the lung parenchyma. Tilt angles between pleura and focal plane were kept as small as possible $\left(<5^{\circ}\right)$. 


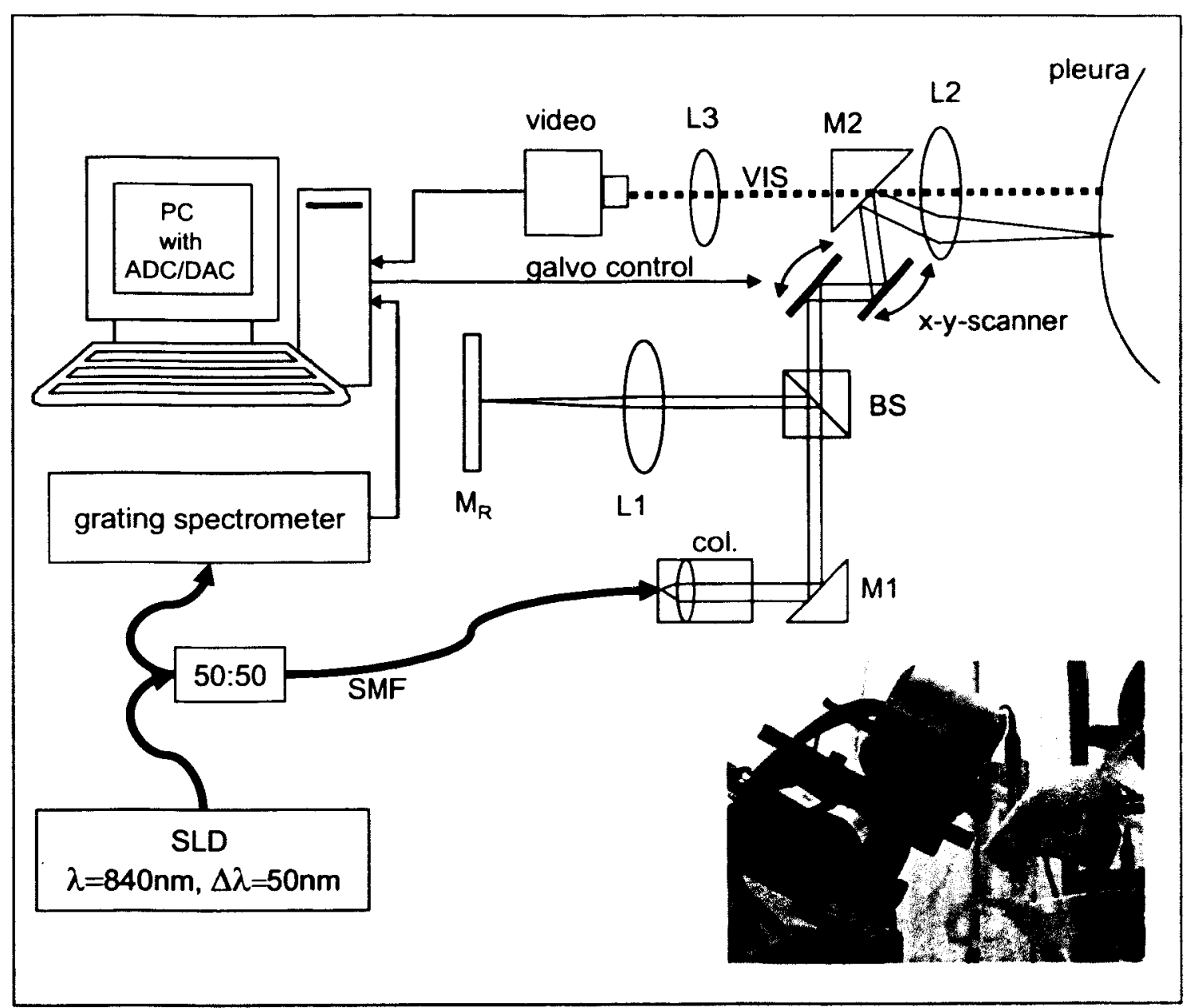

Fig. 1. Sketch of the setup of the Fourier domain OCT system. Insert is a photo of the scanner head mounted on translation stages and a ball socket base aiming at the isolated, ventilated and perfused rabbit lung. SI.D: superluminescence dicode. SMF: single mode fiber, col.: collimator. MI: mirror. BS: 50:50 non-polarizing beamsplitter cube. I. I: reference light focusing lens, MR: reference mirror, M2: dichroic mirror for near infrared/transparent for visible light. L 3 camera lens, $\mathrm{PC}$ with $\mathrm{ADC} / \mathrm{DAC}$ : personal computer with $1.25 \mathrm{MHz} 16$ bit analog to digital conversion for spectral data and digital analog converter for galvanometer control voltage.

\subsection{Image Processing}

The raw spectra are rescaled to wavenumber units by oversampled linear interpolation and spectrally shaped befurce Fourier transformation. The Fourier amplitudes are logarithmized and converted to $x$ bit gresscalc numbers. Ihe resulting image stack (see Fig. 2a) is tilted until the plane of view is parallel to the pleura. 5 slices of the stach at $411 \mu \mathrm{m}$. $44 \mu \mathrm{m}, 48 \mu \mathrm{m}, 52 \mu \mathrm{m}$ and $56 \mu \mathrm{m}$ beneath the strongest pleura reflection are taken for $x-y$ analysu (see $F \mathrm{~g} 2 \mathrm{~h}$ ) $H_{2}$ taking the median along the $z$-axis and a $3 \times 3 \mathrm{kemel}$ median filter in $x-y$ plane the image is despeckled. A hivtigram stretch to the full grey value dynamic range is performed. The resulting image (see Fig. $2 \mathrm{c}$ ) is inverted and filtered br a

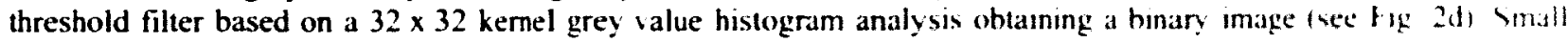
areas are omitted and small holes are filled. The resulting image can be used as a segmentation mavk for separiating wa-ue from air (see Fig. 2f). 


\subsection{Quantification and registration}

The simply connected areas in the binary images are analysed for center of mass coordinates. A uniquely shaped area which can be recognized in every image of a CPAP series is selected and its center of mass is taken as the new reference in each image. After registration of the images to this reference the intersection of the image series is taken and boundary areas are omitted. The number of pixels (real world area $=$ number of pixel $\cdot 16 \mu \mathrm{m}^{2}$ ) for each area is determined in each CPAP step (see Fig. 2e). Areas undergoing fusion or partition from one CPAP level to another are left out for further analysis.

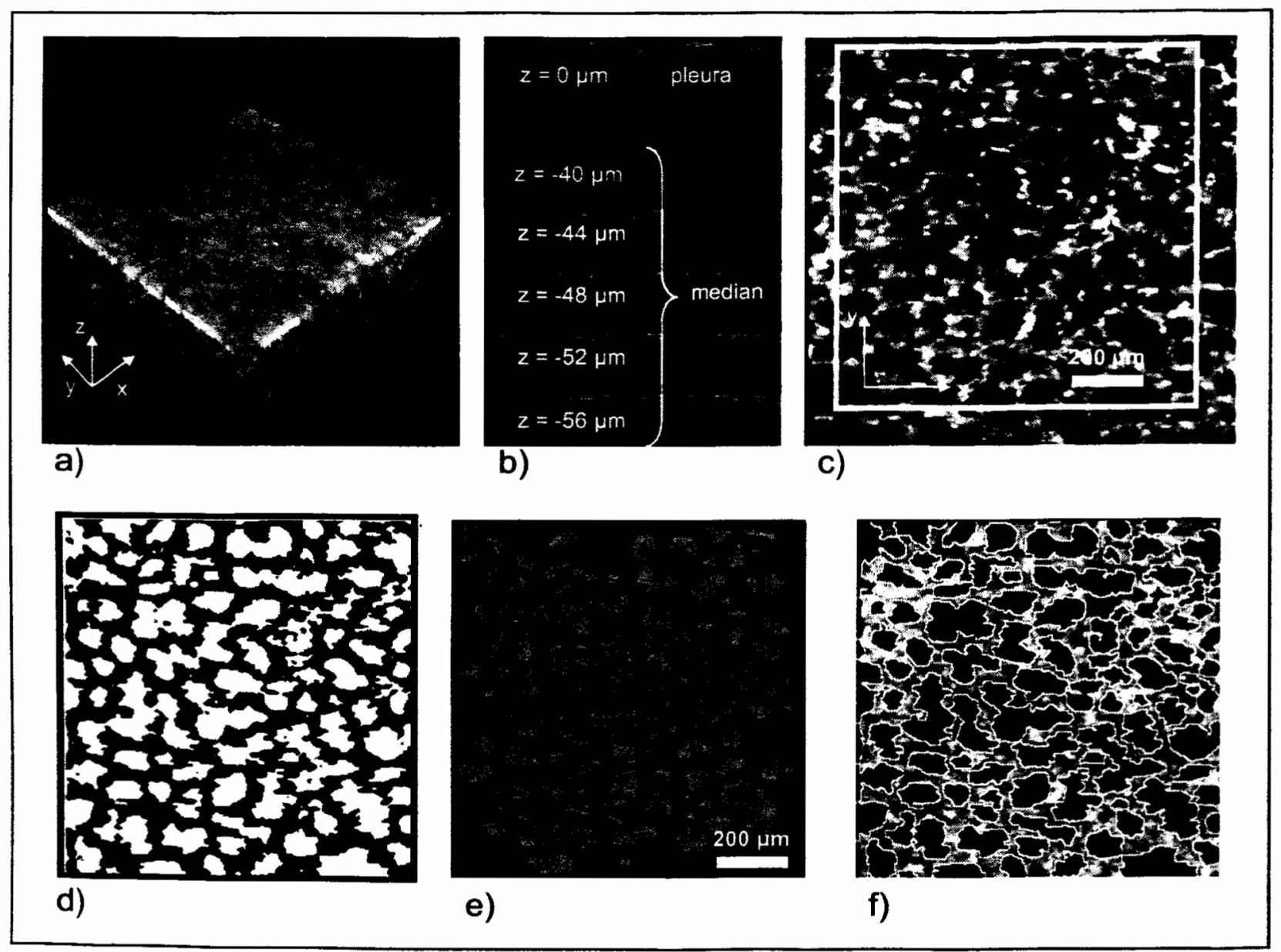

Fig. 2. Illustration of the image processing. a) grey value image stack of isolated rabbit lung at a constant pulmonary airway pressure of 10 mbar (volume rendered view onto pleura). b) five $4 \mu \mathrm{m}$ spaced parallel slices starting $40 \mu \mathrm{m}$ beneath the pleura. c) despeckled median image of the five slices, white box indicates intersection of all images of the series. d) threshold binary image. e) aerated area quantification. f) segmentation lines (white) between air and tissue. c)-f) are shown in the same scale as indicated by the $200 \mu \mathrm{m}$ scale bar. 


\section{RESULTS}

\subsection{Segmented image series}

Simply looking at the OCT image series (Fig. 3) of the alveolar structures with CPAP variation, there is an observable expansion of the aerated areas with rising pressure and correspondingly there is shrinkage with falling pressure. Whereas some alveolar structures stay isolated throughout the pressure variation (circled area in Fig. 3) others fuse or separate (see white arrows in Fig. 3).

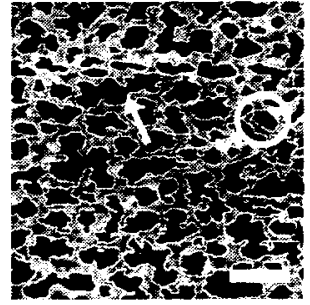

up 5 mbar

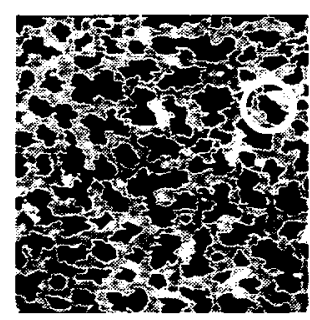

up 25 mbar

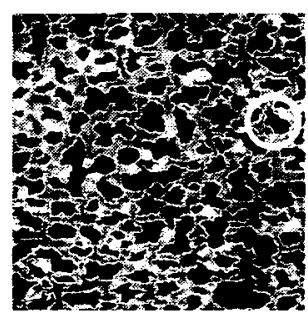

down 5 mbar

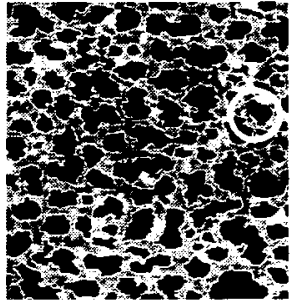

up 10 mbar

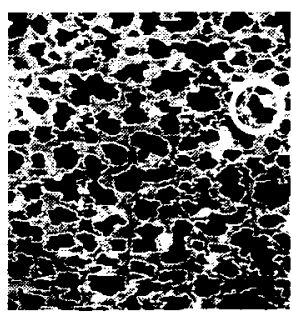

down 20 mbar

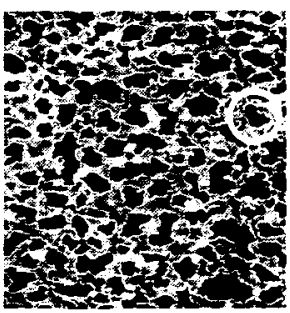

down 4 mbar

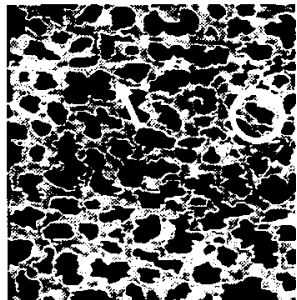

up $15 \mathrm{mbar}$

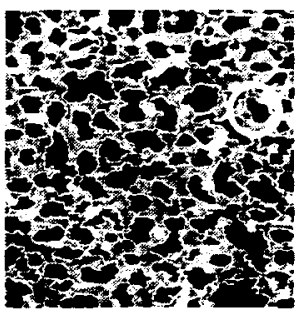

down 15 mbar

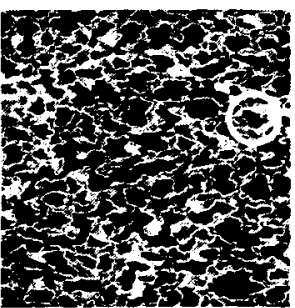

down 3 mbar

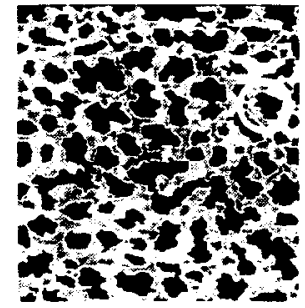

up 20 mbar

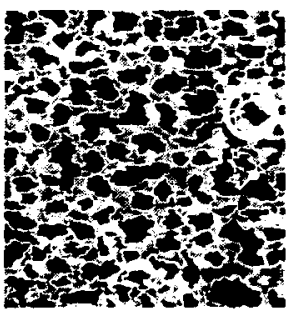

down 10 mbar

Fig. 3. Series of segmented and registered slices of OCT image stacks with $1004 \mu \mathrm{m} \times 1052 \mu \mathrm{m}$ (width $\times$ hight) at different constant pulmonary airway pressures $(u p=$ inflation, down = deflation). White segmentation line added to the processed grey-scale images. White bar indicates $200 \mu \mathrm{m}$ width. White circle picks out the same individual alveolar structure at each CPAP level. White arrow: example of fusion/segmentation of aerated areas during inflation.

\subsection{Quantified cross-sectional areas}

Quantification of pixel area in the segmented aerated areas confirms the qualitative impression of expansion and contraction with pressure. Again there is some heterogeneity. Whereas single alveolar structures show an area-pressure curve (Fig. 4), which has an almost normal hysteresis as expected from the total lung pressure-volume curve, the median curve (Fig. 6) does not allow speaking of a normal hysteresis. The total expansion at 25 mbar reaches more than $+400^{\circ} \%$ of the size at 3 mbar. 


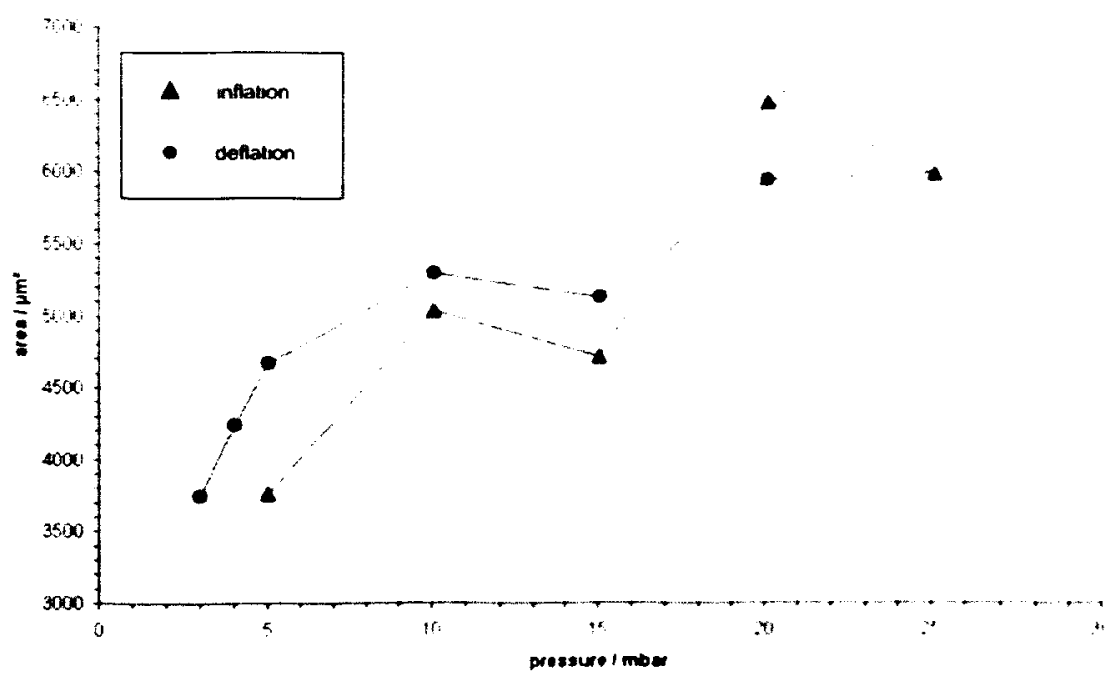

Fig. 4. Diagram showing cross-sectional area of one individual alveolar structure versus $(P \wedge P$. The data is derived from the areas circled in Fig. 3.

In order to check whether the expansion is isotropic, alveolar structures were selected which allowed quantification of all three perpendicular planes. A representative example is shown in Fig. 5. Although there is some deviation between the three planes, there is no evidence for a general difference in the nature of deformation between $x-y, y-z$ and $x-y$-planes.

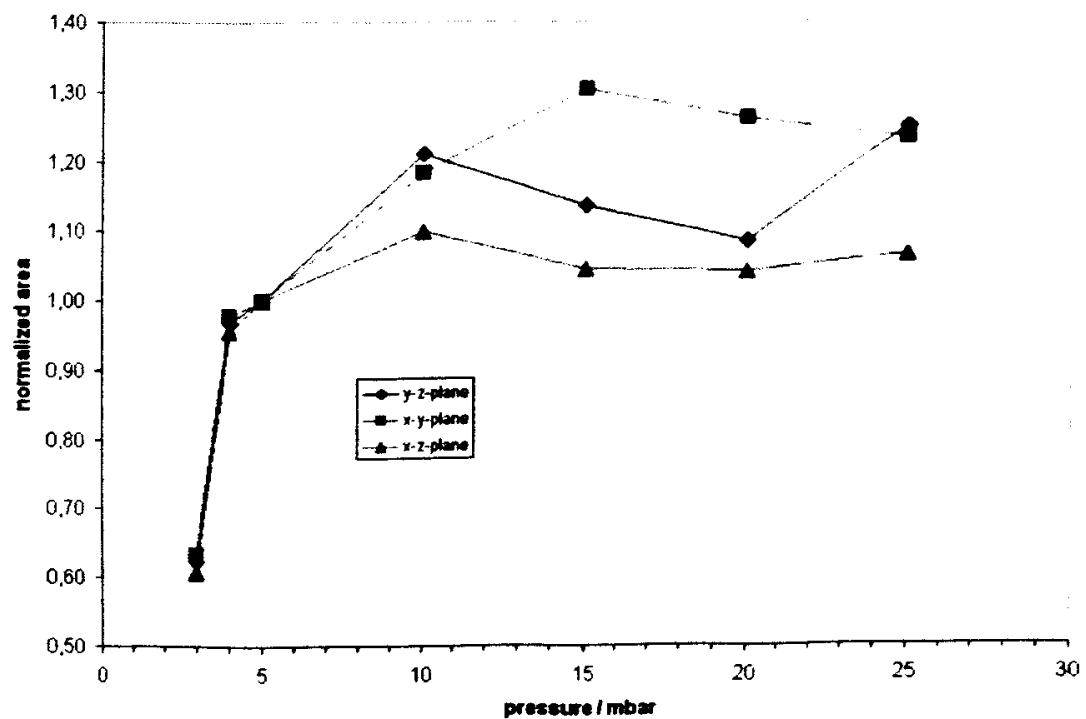

Fig. 5. Plot showing the normalized areas of the three perpendicular planes of one alveolar structure at different CPAP levels. 


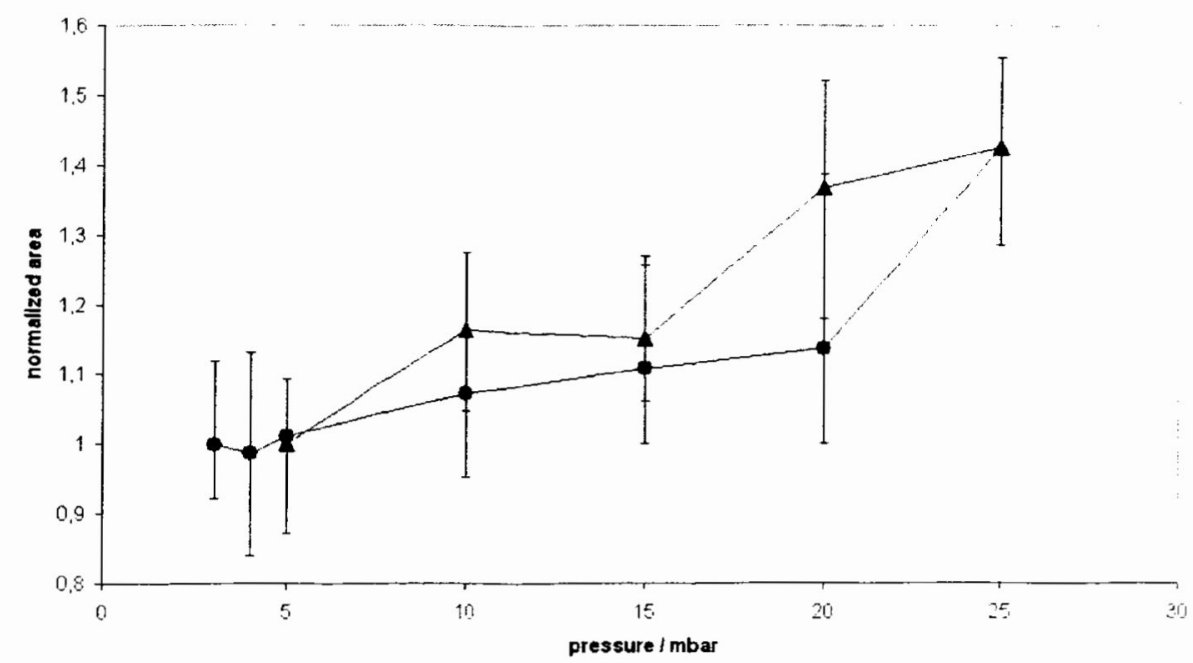

Fig. 6. Diagram showing median value of all analysed areas normalized to their 5 mbar inflation value. Note that there is a strong variation in normalized areas at each CPAP level, but still an overall trend.

\subsection{Inflation rate independent from size}

If one considers the alveolar structures as interconnected balloons, the surface tension could lead to the effect that structures with initially higher volume expand on the cost of smaller volumes. In order to test this hypothesis, the sizes of all analysed areas of one pressure where plotted versus the areas of the same structures at the next CPAP level. At all CPAP levels (see Tab. 1) there was a linear dependency with a coefficient of determination above 0.95 of the new area size on the initial area size (see also Fig. 7) falsifying the stated hypothesis.

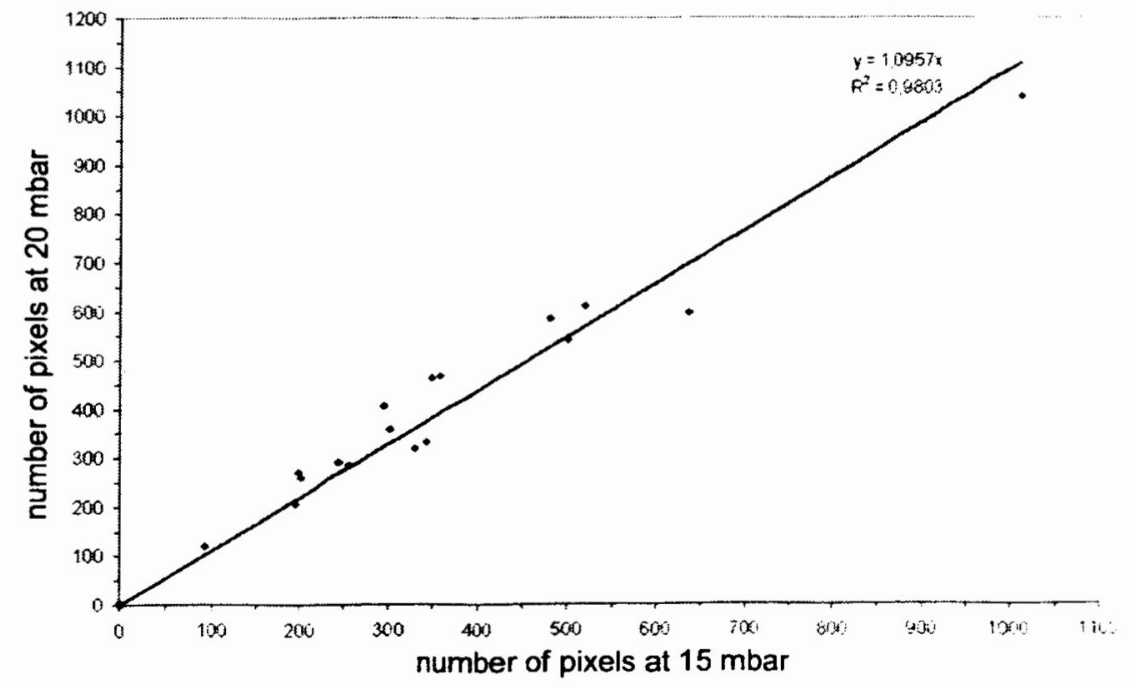

Fig. 7. Plot showing cross-sectional areas at two consecutive CPAP levels. There is a linear dependency, which indicates that there is no systematic dependence of inflation rate on size. 
Tab. I. Linear dependency of cross-sectional areas of two consecutive constant pulmonary airway pressure levels. Refer to Fig. 7. for an example of the corresponding graph.

\begin{tabular}{|c|c|c|}
\hline CPAP-step / mbar & linear factor & Coefficient of determination \\
\hline 5 to 10 & 1.1148 & 0.9557 \\
\hline 10 to 15 & 1.0056 & 0.9784 \\
\hline 15 to 20 & 1.0957 & 0.9803 \\
\hline 20 to 25 & 0.9514 & 0.9954 \\
\hline 25 to 20 & 0.9071 & 0.9929 \\
\hline 20 to 15 & 0.9607 & 0.9849 \\
\hline 15 to 10 & 0.9252 & 0.9864 \\
\hline 10 to 5 & 0.9282 & 0.9864 \\
\hline 5 to 4 & 0.9776 & 0.9861 \\
\hline 4 to 3 & 0.9281 & 0.9893 \\
\hline
\end{tabular}

\subsection{Preserved alveolar structure size during perfusion fixation}

For further analysis the isolated lung was fixated at a CPAP level of $10 \mathrm{mbar}$. To preserve the structures a perfusion fixation with glutaraldehyde solution for $25 \mathrm{~min}$ was performed. During the fixation process six OCT image stacks were acquired at equidistant time points $(t=0,5,10,15,20,25 \mathrm{~min})$. The results are shown in Fig. 8 . The relative size deviates about $2.76 \%(\sigma / \mu)$ along the fixation process without a noticeable trend.

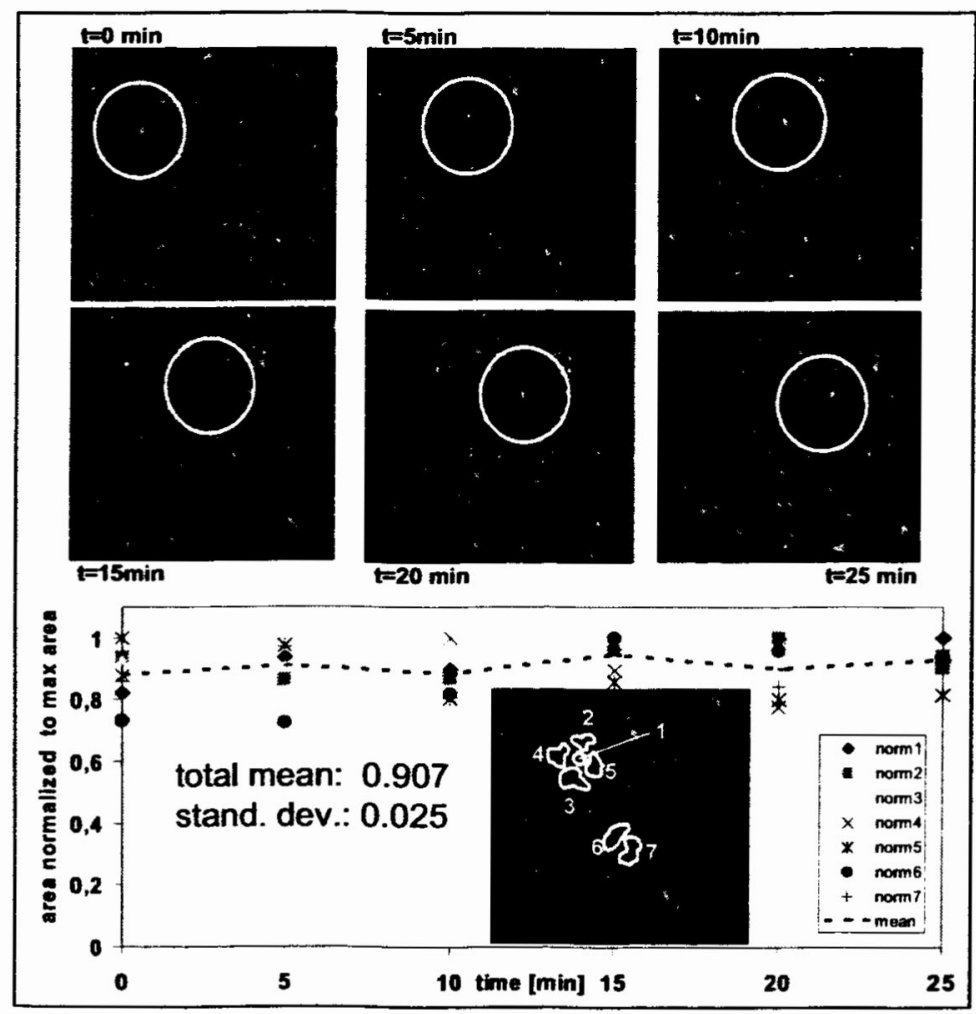

Fig. 8. Six slices were taken from 3D OCT stacks acquired during perfusion with fixative (glutaraldehyde) at different time points $(t=0,5,10,15,20$ and $25 \mathrm{~min}$.). Slices are parallel to the pleura with a distance of $40 \mu \mathrm{m}$. As shown in quantitative plot below there is only minor variation and no systematic shrinkage or expansion during the fixation process. 


\section{DISCUSSION}

With the help of three-dimensional OCT the size of individual alveolar structures can be quantified in the perfused and stepwise inflated isolated rabbit lung. There is a positive correlation with the CPAP level applied and the cross sectional area at $25 \mathrm{mbar}$ is about $140 \%$ of the corresponding area at $3 \mathrm{mbar}$. At least for the isolated lung model and the observed alveolar structures $48 \mu \mathrm{m}$ below the pleura, a rising pressure results in an expansion of alveolar structures

In contrast to dark field microscopy 3D OCT allows to select a fixed depth at which the alveolar walls can be identified unambiguously and separately from structures in the pleura. Although a cross-section parallel to the pleura is the most robust basis for segmentation and quantification, the other two perpendicular planes contain interesting information. too. In some cases a clearly observable lower alveolar wall opposite to the pleura can be detected and quantification of these perpendicular cross sectional areas results in a similar dependence of area on the CPAP level. In this case it is justified to take the third power of the square root of the 2D expansion rates and speak of a pressure-versus-normalized-volumecurve of alveolar structures (the pressure being the constant pulmonary airway pressure - not an alveolar pressure - and the volume being calculated on the basis of cross sectional areas). This relationship could be used as input or verification of numerical calculations on a three-dimensional mechanical model of the lung parenchyma.

In Fourier domain OCT there is always a compromise between depth of focus and transversal resolution. The transwersial resolution in the order of 10-20 $\mu \mathrm{m}$ leads to an overestimation of alveolar wall thickness and conseguently an underestimation of air filled volume. In addition image processing has to be performed before segmentation. mainly to reduce speckle noise. This leads to a wash-out of small structures and may produce artifacts like false air bridges in alveolar walls - or as the opposite case - a closed alveolar wall, where there is a small interconnection in reality. In practice, this makes it difficult to program a fully automated algorithm for area or volume quantification. beciuse: additional fusion and separation take place between CPAP levels. Fortunately, if these structures are manually detected and left out for analysis, there are still enough well behaving structures, which can be quantified along the entire pressure loop. This is only partly true for the two other perpendicular planes. Here, the analysis was limited to about $100 \mu \mu \mathrm{m} \mathrm{of}$ depth beneath the pleura, because of image degrading by multiple scattering at the tissue-air interfaces. This seems 10 be an intrinsic limitation when looking at air-filled parenchyma with optical methods.

Fixation artifacts during a perfusion fixation can be monitored with OCT and quantified to be negligible. This is. however, a very limited statement, because the glutaraldehyde fixation does not fully take out the elastic force of the lung parenchyma. A disconnection of the open trachea from the applied overpressure or even the reopening after additional post fixation overnight still result in a shrinkage of alveolar structure. This, of course. can be monitored with OCT, too.

\section{ACKNOWLEDGEMENTS}

We acknowledge financial support by the German Research Foundation in the context of the protective artificial ventilation research project.

\section{REFERENCES}

1. C. S. Garcia, L. F. Prota, M. M. Morales, P. V. Romero, W. A. Zin, and P. R. Rocco, "Understanding the mechanisms of lung mechanical stress," Braz. J Med Biol. Res 39. 697-706, 2006.

2. J. A. Frank and M. A. Matthay, "Science review: mechanisms of ventilator-induced injury." Crit carc 7. 233-241. 2003.

3. N. E. Vlahakis and R. D. Hubmayr. "Cellular stress failure in ventilator-injured lungs." $4 m$. Respir ( $r u$ ( are Med 171, 1328-1342, 2005.

4. P. Pelosi, S. Crotti, L. Brazzi, and L. Gattinoni, "Computed tomography in adult respiratory distress "yndrome what has it taught us?," Eur Respir. J 9, 1055-1062. 1996.

5. R. D. Hubmayr, "Perspective on lung injury and recruitment: a skeptical look at the opening and collapse stor?." Am. J. Respir. Crit Care Med. 165. 1647-1653. 2002.

6. J. Gil. H. Bachofen, P. Gehr, and E. R. Weibel. "Alveolar volume-surface area relation in air- and saline-filled lungs fixed by vascular perfusion," J. Appl. Physiol 47, 990-1001. 1979. 
7. D. Carney, J. DiRocco, and G. Nieman, "Dynamic alveolar mechanics and ventilator-induced lung injury." Crit Care Med. 33. S122-S128, 2005.

8. N. Hanna, D. Saltzman, D. Mukai, Z. Chen, S. Sasse, J. Milliken, S. Guo, W. Jung, H. Colt, and M. Brenner, "Two-dimensional and 3-dimensional optical coherence tomographic imaging of the airway, lung. and pleura," $J$. Thorac. Cardiovasc. Surg. 129, 615-622, 2005.

9. A. Popp, M. Wendel, L. Knels, T. Koch, and E. Koch, "Imaging of the three-dimensional alveolar structure and the alveolar mechanics of a ventilated and perfused isolated rabbit lung with Fourier domain optical coherence tomography," J Biomed. Opt. 11, 014015-2006.

10. M. G. de Abreu, M. Heintz, A. Heller, R. Szechenyi, D. M. Albrecht, and T. Koch, "One-lung ventilation with high tidal volumes and zero positive end-expiratory pressure is injurious in the isolated rabbit lung model," Anesth. Analg. 96, 220-228, 2003. 